graft status and pure tone audiometry were performed for all the patients and the outcomes were compared.

Results Out of 35 patients in each group, four in the study group and eight in the control group had residual perforation. Graft uptake in case group was $88.57 \%$ and graft uptake in the control group was $77.1 \%$. Result was better in cases that underwent myringoplasty with PRP. Out of 35 patients in case group, audiological improvement (>10 dB) was seen in 31 patients (88.57\%); in the control group of 35 patients, 27 (77.1\%) had audiological benefits.

Conclusion This study shows there is definite benefit by using PRP in myringoplasty. As the PRP can be easily prepared, PRP myringoplasty can be routinely performed.

\section{A0043: Management of Recurrent Collaural Fistula: A Case Report}

Shalima P. S., Shashikant A. Pol, Arjun Dass, Nitin Gupta

Introduction Collaural fistula or cervicoaural fistula is a rare anomaly accounting for less than $8 \%$ of first branchial cleft anomaly. Aberrant development of first branchial cleft may lead to formation of a cervical cyst or sinus in the region of ear.

Case Report We reported a case of a 4-year-old girl who presented with recurrent swelling in right infra-auricular region from 6 months of age. She had undergone incision and drainage of the swelling thrice at various peripheral hospitals over past 3.5 years. On examination, two sinuses were noticed surrounding lobule of right pinna of which one was present posterior to the lobule and second was present just above lobule at lateral most part of conchal cartilage.

Discussion Diagnosis of recurrent infected collaural fistula requires detailed clinical examination for presence of multiple sinuses surrounding the lobule and external auditory canal. In case of nonvisibility of sinus in external auditory canal, microscopic examination should be done. CT sonogram and MRI are useful diagnostic tools for recurrent infected fistulas. Surgical excision of whole tract is the definitive treatment but superficial parotidectomy along with it can reduce chances of recurrence significantly. If more than $30 \%$ of the circumference of external auditory canal is involved then split skin grafting is required for the coverage.

Conclusion Diagnosis of collaural fistula should be kept in mind whenever there is recurrent postaural or infra-aural swelling mainly in pediatric patients. Superficial parotidectomy along with complete excision reduces the recurrence rate. Facial nerve palsy can be a devastating complication of surgery.

\section{A0044: Case Report: Delayed Facial Nerve Paralysis-An Uncommon Complication of Tympanomastoid Surgery Spandana. S. Pardikar}

Introduction Facial nerve paralysis after ear surgery is a troublesome postoperative complication for both patient and the surgeon. Delayed postoperative facial nerve paralysis (DPOFNP) occurs a few days after ear surgery. The cause for this condition is supposed to be reactivation of dormant herpes zoster virus present in geniculate ganglion.
Case Presentation We, hereby, present a case of 25-year-old male, a case of chronic suppurative otitis media atticoantral disease who underwent canal wall down mastoidectomy with tympanoplasty. He presented with complaints of facial nerve paralysis, 7 days after the surgery. Patient had a House-Brackmann grade-3 paralysis. Treatment was initiated with oral corticosteroids and oral acyclovir. Patient was recovered completely after the therapy.

Discussion DPOFNP is an uncommon complication following uneventful tympanomastoid surgery. It is due to mechanical reactivation of HSV-1 virus in geniculate ganglion following mechanical disturbance of chorda tympani or operating in close vicinity to the facial nerve. It can also occur following neuro-otological surgery like acoustic neuroma and vestibular neurectomy, following stapedectomy or cochlear implantation.

Conclusion Patient presenting with facial nerve paralysis a few days after uneventful tympanomastoid surgery should be diagnosed as DOPFNP. The patient should be reassured and given a course of oral corticosteroids and acyclovir. Overall prognosis is good.

\section{A0045: Ancient Schwannoma Mimicking Glomus Jugulare Sreenivas Kamath K.}

Introduction Schwannomas are benign tumors arising from the nerve sheath. Schwannoma of the jugular foramen (JF) is rare. We report a case of ancient schwannoma arising from the JF, the first of its kind to be reported in the literature.

Case Report A 37-year-old male presented with ear pain, pulsatile tinnitus, reduced hearing in the left side since 6 months and ear discharge since 3 months. Past history of ear surgery was done for ear discharge 15 years back. No history of difficulty in speech and swallowing, nasal regurgitation, facial deviation, hemifacial spasms, or dizziness was reported.

On examination: left EAC-reddish polyp was present, did not bleed or pain on touch. Post aural scar was present. Right ear: normal. Audiological testing was suggestive of moderate conductive hearing loss on left and minimal hearing loss on right. There were no obvious neurological deficits on examination.

Investigations: HRCT showed skull base isodense lesion with extension into jugulare foramen-glomus jugulare (GJ). Contrast MRI well defined extra-axial, soft tissue enhancing mass in relation to the left $\mathrm{CP}$ angle involving petrous part of temporal bone with extension into the middle ear and external ear with salt and pepper appearance-GJ/ Nerve sheath tumor. MRA showed encasement of the ICA by the lesion with no obvious feeders.

Treatment: patient underwent left side transmastoid transtemporal excision of the tumor. Intraoperatively ECV and IJV were ligated. Facial nerve was identified and transposed. Tumor mass completely removed in peacemeal, reconfirmed with endoscopic evaluation. Defect was sealed using abdominal fat graft. Postoperative grade-4 facial palsy noted. HPE: spindle cells arranged in sheets having elongated nuclei 